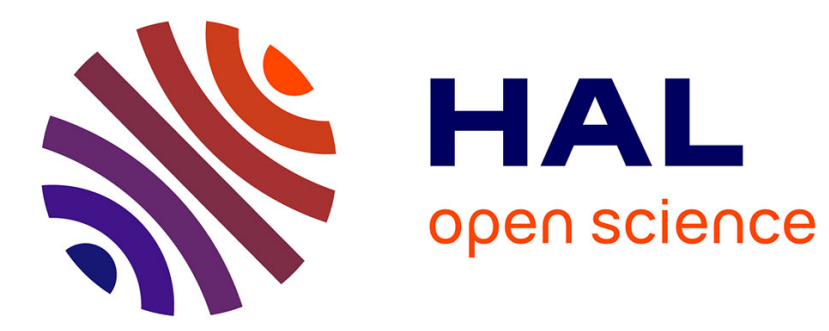

\title{
Decoupling the Eye: A Key toward a Robust Hovering for Sighted Aerial Robots
}

\author{
Augustin Manecy, Raphaël Juston, Nicolas Marchand, Stéphane Viollet
}

\section{To cite this version:}

Augustin Manecy, Raphaël Juston, Nicolas Marchand, Stéphane Viollet. Decoupling the Eye: A Key toward a Robust Hovering for Sighted Aerial Robots. Advances in Aerospace Guidance, Navigation and Control, Springer, pp.317-336, 2013, 978-3-642-38253-6. 10.1007/978-3-642-38253-6_20 . hal00819744

\section{HAL Id: hal-00819744 \\ https://hal.science/hal-00819744}

Submitted on 2 May 2013

HAL is a multi-disciplinary open access archive for the deposit and dissemination of scientific research documents, whether they are published or not. The documents may come from teaching and research institutions in France or abroad, or from public or private research centers.
L'archive ouverte pluridisciplinaire HAL, est destinée au dépôt et à la diffusion de documents scientifiques de niveau recherche, publiés ou non, émanant des établissements d'enseignement et de recherche français ou étrangers, des laboratoires publics ou privés. 


\title{
Decoupling the eye: a Key toward a Robust Hovering for sighted Aerial Robots
}

\author{
Augustin Manecy, Raphaël Juston, Nicolas Marchand and Stéphane Viollet, \\ member IEEE
}

\begin{abstract}
Inspired by natural visual systems where gaze stabilization is at a premium, we simulated an aerial robot with a decoupled eye to achieve more robust hovering above a ground target despite strong lateral and rotational disturbances. In this paper, two different robots are compared for the same disturbances and displacements. The first robot is equipped with a fixed eye featuring a large field-ofview (FOV) and the second robot is endowed with a decoupled eye featuring a small FOV (about $\pm 5^{\circ}$ ). Even if this mechanical decoupling increases the mechanical complexity of the robot, this study demonstrates that disturbances are rejected faster and computational complexity is clearly decreased. Thanks to bio-inspired visuo-motor reflexes, the decoupled eye robot is able to hold its gaze locked onto a distant target and to reject strong disturbances by profiting of the small inertia of the decoupled eye.
\end{abstract}

Part of this paper reprinted from "Bio-Inspired Hovering Control for an Aerial Robot Equipped with a Decoupled Eye and a Rate Gyro" by A.Manecy, S. Viollet and N. Marchand, which appeared in Intelligent Robots and Systems (IROS), 2012 IEEE/RSJ International Conference on. 2012 IEEE [1].

Manecy Augustin

GIPSA-lab laboratory, Control Systems Dept., SySCo team, CNRS-Univ. of Grenoble, ENSE ${ }^{3}$ BP 46, 38402 St Martin d'Hères Cedex, France and the Aix-Marseille Université, Biorobotic Dept. of ISM, CNRS, ISM UMR7287, 13288, Marseille cedex 09, France, e-mail: augustin.manecy@univamu.fr

Raphaël Juston

Aix-Marseille Université, Biorobotic Dept. of ISM, CNRS, ISM UMR7287, 13288, Marseille cedex 09, France, e-mail: raphael.juston@univ-amu.fr

Nicolas Marchand

GIPSA-lab laboratory, Control Systems Dept., SySCo team, CNRS-Univ. of Grenoble, ENSE ${ }^{3}$ BP 46, 38402 St Martin d'Hères Cedex, France e-mail: nicolas.marchand@gipsa-lab.fr

Stéphane Viollet

Aix-Marseille Université, Biorobotic Dept. of ISM, CNRS, ISM UMR7287, 13288, Marseille cedex 09, France, e-mail: stephane.viollet@univ-amu.fr 


\title{
Acronyms
}

\author{
FOV Field Of View. \\ rVOR Rotational Vestibulo-Ocular Reflex. \\ tVOR Translational Vestibulo-Ocular Reflex. \\ VFR Visual Fixation Reflex. \\ ZSL Zero-Setting System. \\ VFL Visual Feedback Loop. \\ D-EYE Decoupled eye system. \\ F-EYE Fixed eye system.
}

\section{Introduction}

Several methods have been developed during the last few years which have enabled UAVs to fly increasingly autonomously (to perform automatic taking off and landing, etc.). Even if it is possible to estimate the attitude of an UAV using only an IMU, this last cannot be used to estimate the position without drifts. As a consequence, IMUs are combined with other sensors to provide both an attitude and pose estimations. Some ways consist in the fusion of the IMU and the GPS to avoid drifts in estimation, as in [2] or [3] and more recently [4]. And most of the strategies used for this purpose were based on a combination of vision sensors and Inertial Measurement Units. For instance, [5] used a trajectometric system of measurement to determine the position and orientation of a quad-rotor. Despite the high frame rate and the good resolution of this system, the robot cannot be said to be fully autonomous because of the off-board data processing system on which it depends. A similar system involving the use of a CCD camera was developed by [6]. Another strategy consisted of using active markers placed in the environment (one under the robot and one in front of it), as described by [7] to assess the robot's attitude and position. Along similar lines, using an embedded camera and a different set of geometrical markers (five), [8] provided a robot with an accurate means of estimating both its position and its orientation. A similar task was also accomplished in a study by [9], using active markers and a simple visual sensor borrowed from a remote Wii control (Wiimote). Other methods based on the use of optical flow have been presented, as in [10], for determining the altitude, position and speed of a robot flying above a road with specific geometric characteristics. In all these approaches, the robot's position was estimated via visual sensors, as in [5] combined with an embedded inertial measurement unit. In the latter case, the gyrometer's bias was classically compensated for by means of accelerometers (see [11]).

To make UAVs more autonomous, several methods relying on SLAM algorithms use monocular camera to navigate in an unknown GPS-denied environment ([12]). Other SLAM methods use laser rangefinder as an active optical sensor, combined with a laser mirror for height estimation as in [13] or [14]. SLAM was also per- 
formed by merging both information from a camera and from a laser rangefinder [15].

We focused our work on the advantages provided by a decoupled eye embedded onboard a UAV. In previous robotic studies, a decoupled eye is classically used to track a target and compensate for the UAV displacements around this target. This objective was achieved for example in [16], where a target was successfully tracked (from an autonomous helicopter). The positions and attitude of the helicopter were estimated by means of a data fusion algorithm between a GPS, a magnetometer and an IMU. We demonstrate here that a decoupled eye (with a narrow field of view) could be used not only to track a target, but also to estimate the position and the attitude of a UAV using only the retinal error, the orientation of the eye with respect to the robot and a rate gyro.

In this way, the hovering robot presented here was assumed to have an "eye" with a controlled rotation relative to its "body". In addition, the eye was taken to have a narrow FOV of only a few degrees (a kind of fovea). The visual sensor is able to rotate and thus to change the gaze direction (the line of sight). As described in [17], a fovea equipped with a gaze control mechanism of this kind is a considerable step forward in the computational modeling of vision, where visual and control systems with many degrees of freedom have to solve difficult problems without gaze control mechanisms. The present additional degree of freedom mimics the characteristic mechanical decoupling between the eye and body of many animals, such as the hoverfly. The present robot's eye can be said to be a sensitive, accurate visual position-sensing device (PSD) [18], which is able to detect the position of an edge (or a bar) within its very small field of view (here, $F O V= \pm 5^{\circ}$ in comparison with the FOV of more than $50^{\circ}$ in the case of the robots used in previous references). This sensor's performances in an edge-detection task were a 40-fold improvement in terms of the resolution, as compared with the previous interphotodiode angular resolution [19]. The visual sensor in question can therefore be said to be endowed with hyperacuity [20]. For further details about the performances (i.e., the accuracy and calibration) of this hyperacute visual PSD, see [21] and [19]. Gaze stabilization is a difficult task because the eye control system must compensate both quickly and accurately for any sudden, untoward disturbances caused by the vagaries of the supporting head or body. This finely adapted mechanism is way beyond what can be achieved in the field of present-day robotics. The only information available onboard the present robot, in line with what occurs in its natural counterparts, is the inertial measurement provided by the biased rate gyro and the eye-in-body orientation provided by a hall effect sensor.

The robot with a decoupled eye is presented in the next section, along with its nonlinear dynamic model. In Section 3, the original nonlinear observer used to estimate the robot's position, attitude (roll axis only) and unbiased rotational speed is presented. The eye control system and the robot's overall control system based on this observer are then described. In section 4, the advantages of implementing a feedforward gaze control process are discussed and detailed comparisons are made between the behavior of the simulated robots with and without a decoupled eye. A comparison between a fixed eye robot endowed with a large FOV and the decoupled 
eye robot with a narrow field of view is equally done to demonstrate advantages provided by the decoupling.

\section{System overview}

In this section, we describe the aerial robot and the computational resources embedded on-board. We describe also the nonlinear model of the robot which was used to simulate the different scenarios.

\subsection{The twin engine hovering robot}

In this part, we introduce the robot and its equipment. We explain the different objectives and describe our bio-inspired approach.

\subsubsection{The robot's hardware}

As shown in the CAD in figure 1, the twin-engine aerial robot we have designed will have three degrees of freedom (a rotation around the horizontal axis, $\theta_{r}$, a right and left translation, $X$, and an eye rotation $\theta_{e r}$ with respect to the robot's body). Thanks to a mechanical decoupling between the eye and its mechanical support (the head), the eye can rotate freely in the robot's frame. In addition, the eye's orientation can be finely controlled by means of an extremely compact, fast and accurate servo (MKS). The robot, which will weigh only about 150 grams in all, will be completely autonomous, thanks to its embedded computational resources and the on-board power supply described as depicted in figure 2 .

As shown in figure 4, the HyperRob's roll angle $\theta_{r}$ can be controlled by applying a differential rotational speed to the propellers. The robot was assumed in the present simulations to fly at a constant altitude $H$. This assumption is not restrictive, since it is known that the altitude can be decoupled from the roll and lateral movements in systems of this kind, which belong to the same class as aircraft capable of performing planar vertical take- off and landing ([10], [9] or [6]). As the flying robot is under-actuated, its position $X$ on the horizontal axis is controlled by adjusting its attitude around the roll axis. The robot is therefore in a state of equilibrium when

$\theta_{r}=0^{\circ}$. In this study, the robot was assumed to be hovering above a target on the ground. 


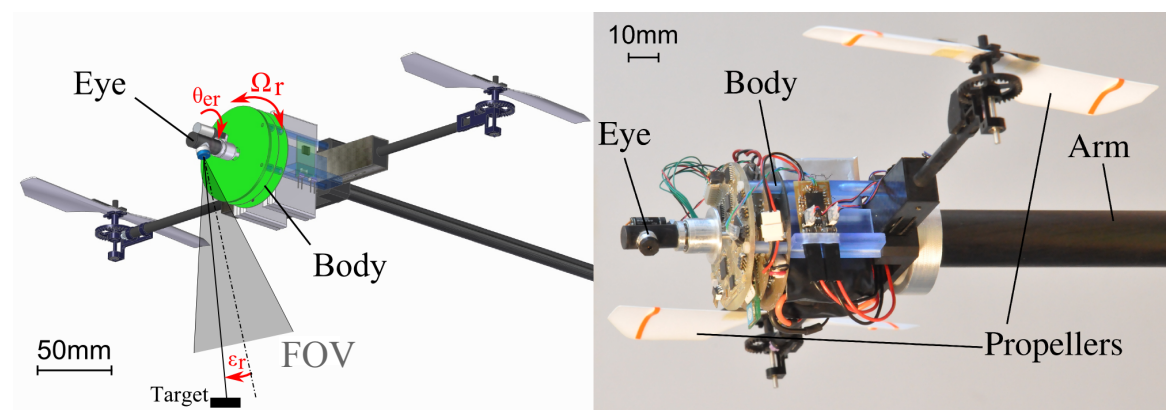

Fig. 1 (a) CAD of the 150-grams micro-air vehicle, called HyperRob, in which a fast micro servo (MKS servo) controls the orientation of the eye (angle $\theta_{e r}$ ) relative to that of the body. The orientation of the robot around the roll axis (angle $\theta_{r}$ ) is controlled by applying a differential rotational speed to the propellers. The robot itself was mounted here at the tip of a rotating arm allowing to move freely in the horizontal plane. (b) Picture of the HyperRob robot fixed at the tip of an arm rotating in the azimut plane.

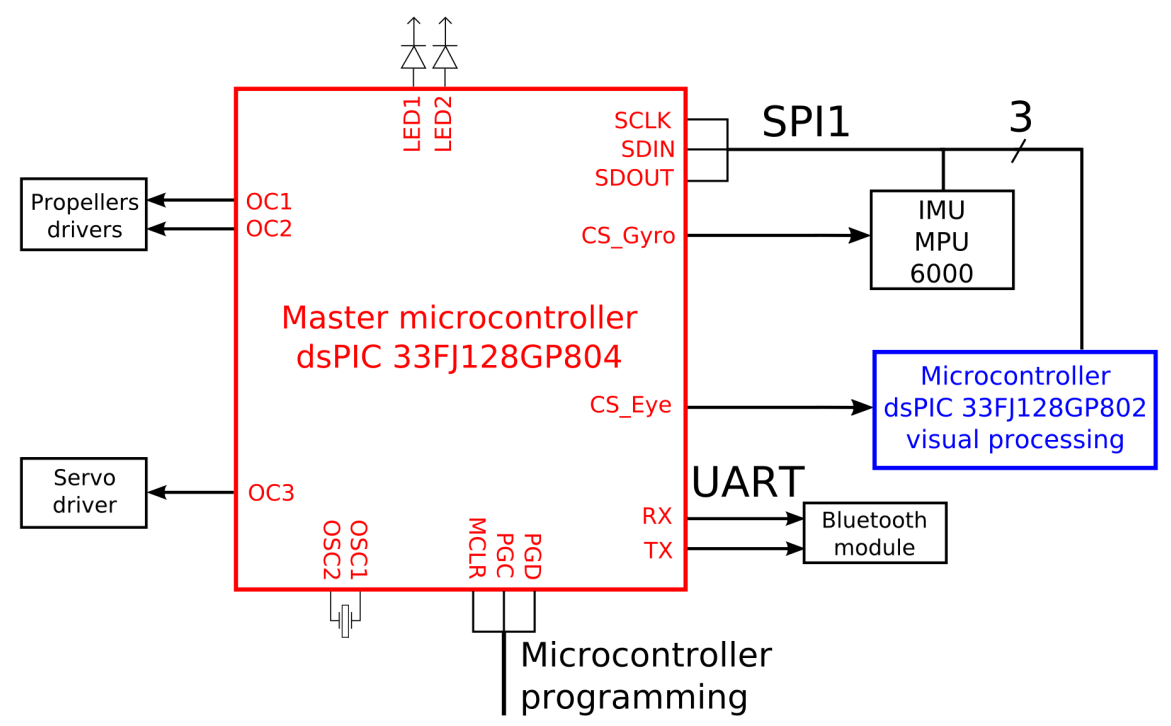

Fig. 2 Simplified scheme of the embedded electronics. The robot is equipped with two Microchip dsPIC microcontrollers (16 bits). The main microcontroller (dsPIC 33FJ128GP804) runs a multirate Simulink-based program, which is in charge of the main control tasks. A secondary microcontrollers (dsPIC 33FJ128GP802) is used to process the visual signals of the eye. The main microcontroller sends the set point specifying both the eye's angular position and the throttle of the two propellers via PWM signals. The autopilot uses solely the rate gyro (roll axis) of an inertial measurement unit (MPU6000) and digital signals from the visual processing unit. A Bluetooth wireless device connected to the UART peripheral can be used by the operator to log data received from the robot and to send the robot data and the start/stop instructions.

\subsubsection{The bio-inspired approach}

Setting our hovering control strategy in a bio-inspired minimalistic framework meant that the objective was to stabilize the under-actuated hovering robot using 
only a drifting rate gyro and an eye with a narrow field of view. As can be seen from Figure 3, our robot has many points in common with the fly:

- A rate gyro: the fly has two gyroscopic halteres organ measuring its body's angular speed in the three degrees of rotation (pitch, roll and yaw) [22], whereas the robot is equipped with a classical MEMS rate gyro.

- An optical position sensing device: the fly's compound eye is able to locate a contrasting target placed in a small frontal part of the visual field [23], [24], while the robot is equipped with an eye endowed with hyperacuity [25], with which it can accurately locate the targets occurring in its small FOV.

- A neck: the fly has no less than 23 pairs of muscles with which to control its head's orientation [26]. The robot has a decoupled eye, which is actuated by means of a tiny position servomotor (MKS).

- A proprioceptive sensor in the neck: the fly has prosternal organs consisting of a pair of mechanosensitive hair fields located in the neck [27], while the robot is equipped with a contactless magnetoresistive sensor measuring the orientation of the eye relative to the head.

- A gaze stabilization: in the freely flying sand wasp, active gaze stabilization mechanisms prevent the incoming visual information from being affected by disturbances, such as large body rotations around the roll axis [28]. The robot therefore uses two bio-inspired oculomotor reflexes to compensate for its own body movements.

Fig. 3 Similarities between the fly (a) and the hovering robot with a decoupled eye (b). These two dynamic underactuated systems are able to measure their body's rotational speed $\Omega_{r}$ by means of a rate gyro (in the case of the robot) and halteres (in that of the fly) and to locate a contrasting target $\theta_{t}$ placed in a small part of their FOV. The fly has no less than 23 pairs of muscles in its neck, with which to stabilize its gaze $\theta_{g}$, whereas the robot controls the angular position of its eye $\theta_{e r}$ by means of a small servomotor. Here, the fly and

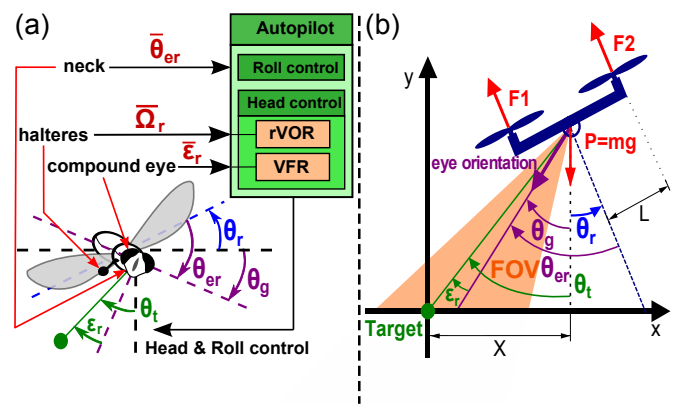
the robot are hovering over a ground target.

In what follows, we have used the following notations:

- $\theta_{t}$ : the angular position of the target in the inertial frame.

- $\theta_{r}$ : the robot's roll angle.

- $\theta_{e r}$ : the angle between the eye and the robot in the robot's frame. This angle is mechanically constrained to a maximum value: $\left|\theta_{e r}\right|<\theta_{e r-M A X}$.

- $\theta_{g}$ : the angular position of the gaze in the inertial frame $\theta_{g}=\theta_{e r}+\theta_{r}$. 


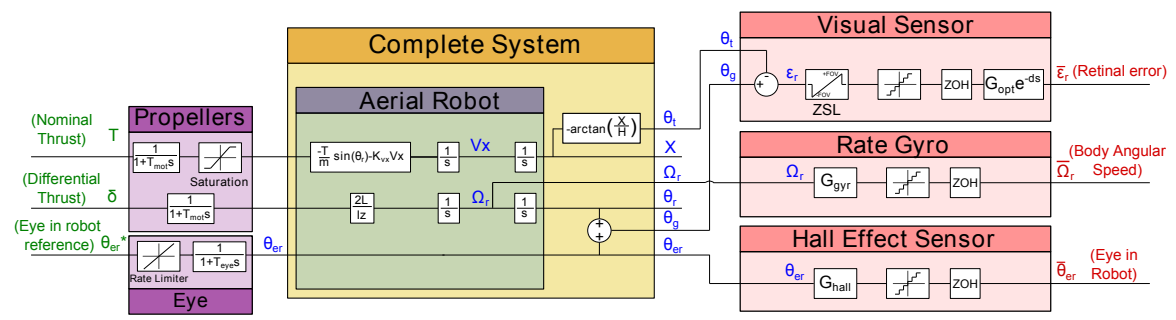

Fig. 4 Block diagram of the complete system. The robot is equipped with a rate gyro, which measures the rotational speed around the roll axis and a decoupled eye locked onto a distant target. Green variables are the control input signals, blue ones are the main physical variables of interest and red ones are the measured values.

- $\varepsilon_{r}$ : the retinal error defined by $\varepsilon_{r}=\theta_{g}-\theta_{t}$.

- $X$ : the position of the robot along the horizontal axis in the inertial frame.

- $V_{x}$ : the speed of the robot on the horizontal axis in the inertial frame.

- $Y$ : the position of the robot on the vertical axis in the inertial frame. $Y$ was assumed to be constant $(Y(t)=H \quad \forall t)$.

- $\Omega_{r}$ : the robot's rotational roll speed.

Estimated values are denoted by an additional hat (e.g., $\hat{\theta})$, reference values by a star (e.g., $\left.\theta^{\star}\right)$ and measured values by a bar (e.g., $\bar{\theta}$ ).

\subsection{Non-linear model of the hovering robot}

A classical nonlinear dynamic model was adopted for the robot in the inertial frame:

$$
\left\{\begin{aligned}
\dot{V}_{x} & =\frac{-\left(F_{1}+F_{2}\right) \sin \left(\theta_{r}\right)}{m}-K_{v x} V_{x} \\
\dot{X} & =V_{x} \\
\dot{\Omega}_{r} & =\frac{L\left(F_{2}-F_{1}\right)}{I_{z}} \\
\dot{\theta}_{r} & =\Omega_{r}
\end{aligned}\right.
$$

where $L$ is the distance between the center of the robot and the propellers, $I_{z}$ is the inertial momentum around the roll axis, $F_{1}$ and $F_{2}$ are the thrust generated by propellers 1 and 2, respectively, and $K_{v x}$ is the flapping coefficient, which is assumed to be constant.

An internal speed loop makes the robot's rotational speed $\left(\Omega_{r}\right)$ exactly follow the rotational speed set points $\left(\Omega_{r}^{\star}\right)$ dictated by the attitude controller (see 3.4). To control the rotational speed $\left(\Omega_{r}\right)$, it was assumed that the propellers are controlled directly via the thrust value. The control input signal to a propeller is there- 
fore composed of a nominal thrust and a differential thrust. The nominal thrust $\left(T^{\star}\right)$ counteracts the gravity and the differential thrust $\left(\delta^{\star}\right)$ generates the torque responsible for the roll rotation. The propeller's control input signals are defined by $F_{1}^{\star}(p)=T^{\star}(p)-\delta^{\star}(p)$ and $F_{2}^{\star}(p)=T^{\star}(p)+\delta^{\star}(p)$. The propellers' dynamics were assumed to correspond to a first order system with a time constant equal to $\tau_{\text {mot }}$ (see table 2).

Figure 4 shows the complete model of the robot including the propellers, the rate gyro, the visual sensor and the angle sensor (used to measure the eye-in-robot angle $\theta_{e r}$ ). In the dynamic simulations of the robot, which were computed with the Matlab/Simulink environment, the set of parameters presented in table 1 were used.

\section{Gaze, attitude and position stabilization}

In this section we present the hierarchy and the interconnections between the different controllers. The stabilization problem was divided in three independent stabilization loops. As the robot is under actuated, a first low level controller allows to track the rotational speed reference. This reference is yielded by the high level position and attitude controller which use the roll angle to achieve the wished position. And finally a reflex based controller allows to lock the target in the small FOV of the eye thanks to adjusting the eye-in-robot angle.

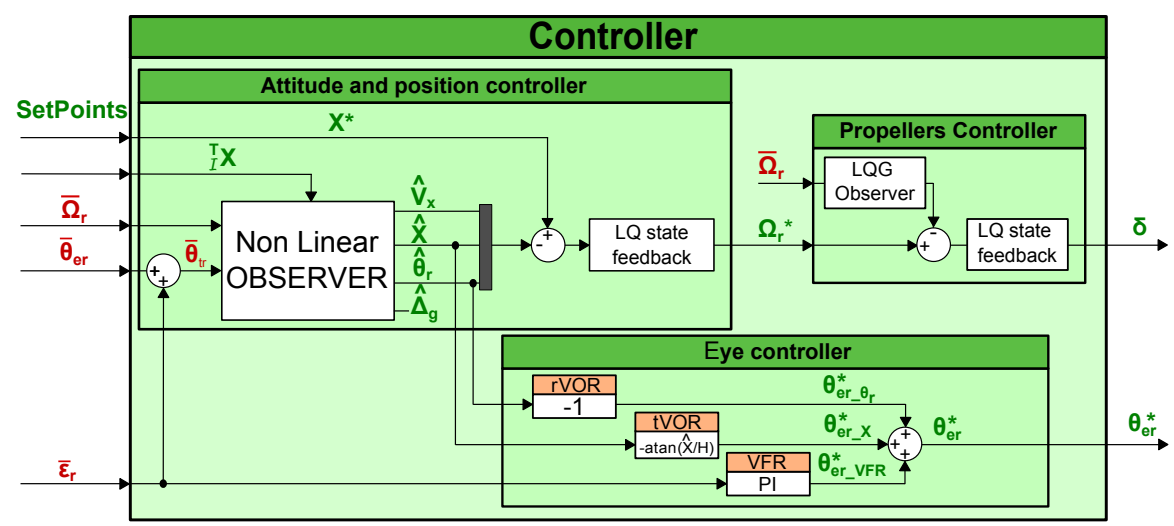

Fig. 5 Block-diagram of the controller. The nonlinear observer determines the robot's attitude on the sole basis of its angular speed and the eye orientation measurements. The eye controller is composed of three oculomotor reflexes (ORs), which are in charge of keeping the eye locked on the target whatever translational or rotational disturbances may occur. Measured (simulated) signals are presented in red and control input signals in green. 


\subsection{The nonlinear observer}

In the classical approach, the attitude estimation problem is solved thanks to a nonlinear complementary filter ([29]) using both the accelerometers and the rate gyros. In this case, the rate gyros offer good information for estimating robot attitude during aggressive maneuvers. And the accelerometers are used as an inclinometer relative to the gravity acceleration allowing to compensate rate gyro bias. In this work, the estimation problem is solved without using classical IMU, and the complete attitude, position relative to target and rate gyro's bias are obtained with only two measurements.

The nonlinear observer is the cornerstone of our hovering control strategy, because it delivers the inputs of the position and attitude controller and especially for the VOR (see 3.2). So on the basis of two measurements which are the rotational speed $\bar{\Omega}_{r}$ and the retinal error $\bar{\varepsilon}_{r}$, the nonlinear observer yields an estimate for the linear speed $V_{x}$, the position $X$, the roll angle $\theta_{r}$ and the rate gyro's bias $\Delta_{g}$ :

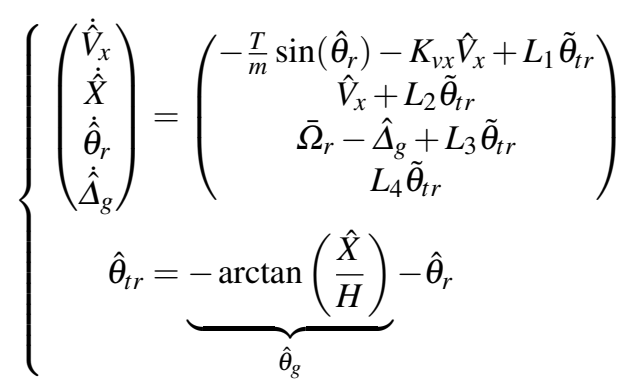

Where $\tilde{\theta}_{t r}=(\underbrace{\bar{\theta}_{e r}+\bar{\varepsilon}_{r}}_{\bar{\theta}_{t r}}-\hat{\theta}_{t r})$

We introduce here the angle $\theta_{t r}$ which corresponds to the orientation of the target in the robot's frame. This new angle improves our previous work ([1]), where it was assumed that $\theta_{e r}=\theta_{t r}$ and that the observer's innovation term was $\tilde{\theta}_{e r}=\bar{\theta}_{e r}-\hat{\theta}_{e r}$. As a consequence, the retinal error was used to improve the estimation of the angular position of the target. This modification improves the quality of the estimations and leads to a better roll stabilization (see figure 9.b) in comparison to our previous work.

We decided to implement a nonlinear observer because of the strongly nonlinear equations giving the evolution of the linear speed $V_{x}$ and the robot's position $X$ (see equation (2)). Non-linearities in $\hat{V}_{x}$ give more accurate estimations during transient responses, whereas non linearities in $\hat{\theta}_{e r}$ result in non steady state error in the position estimates. Observer gain $L=\left(L_{1} L_{2} L_{3} L_{4}\right)^{T}$ was tuned by applying the classical LQG method to the system linearized around the origin. 


\subsection{The Eye controller}

This controller is essential for the robot, because it makes the robot's gaze locked onto the target on the ground. This gaze control loop has to be really fast and accurate to provide to the eye the capacity to reject disturbances which the robot without decoupled eye could not reject (due to its bigger inertia). This fast controller is achieved by merging three complementary oculomotor reflexes which combine feedforward and feedback control:

- A rotational vestibulo ocular reflex, called the rVOR, yields the output signal $\theta_{e r_{-} \theta_{r}}^{\star}$, which is simply equal to the opposite of the estimated roll angle $\hat{\theta}_{r}$.

- A translational vestibulo ocular reflex, called the tVOR, which depends on the estimated robot's linear position $\hat{X}$, assuming that the robot's altitude is known. This reflex compensates for any translation applied to the robot's body by producing the output signal $\theta_{e r_{X} X}^{\star}$, which contributes to keeping the eye locked onto the target.

- A visual fixation reflex (VFR), where the visual feedback loop cancels the retinal signal error $\varepsilon_{r}$ by controlling the eye's orientation $\theta_{e r}$ via the control input signal $\theta_{\text {er_VFR }}^{\star}$ (see figure 6).

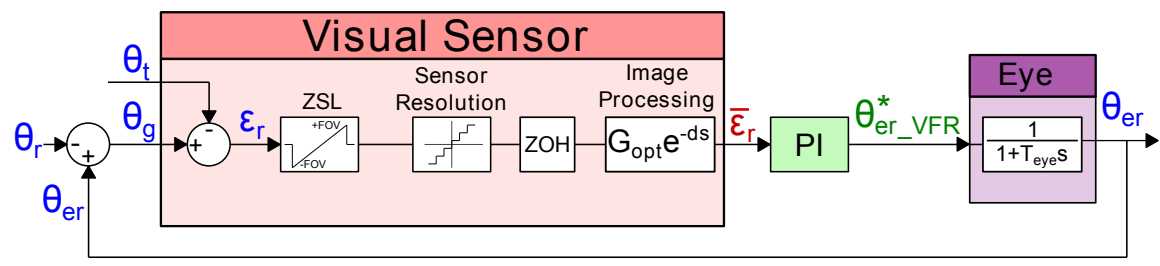

Fig. 6 Block diagram of the visual feedback loop used to implement the visual fixation reflex (VFR). A Proportional-Integral controller cancels the retinal error $\varepsilon_{r}$. The ZSL "Zero-Setting Limiter" serves here to prevent runaway of the eye if the target is lost. $G_{\text {opt }}$ is the static gain of the visual sensor and $d$ is a pure delay introduced by the image processing.

It is worth noting that the ZSL function shown in figure 6 clamps the retinal error back to zero whenever the latter tends to become higher (or lower) than a specified positive (or negative) level. This ZSL, which was used in previous studies ([21], [30]) serves the same purpose as the limiter block used to model the inhibition of the smooth pursuit reflex whenever the position error goes beyond a fixed threshold [31], [32].

\subsubsection{Visual Fixation Reflex (VFR)}

The VFR depicted in figure 6 plays the most important role because it makes the robot: 
- reject any lateral disturbances (gusts of wind),

- correct unmodelled dynamics in the feedforward terms,

- follow a moving target with a high level of accuracy.

The VFR controller is a simple proportional-integral controller, which keeps the retinal error close to $0^{\circ}$ by producing a reference angle $\left(\theta_{\text {er_VFR }}^{\star}\right)$. When designing the PI controller, the pure delay resulting from the computational time and the visual sensor's latency was approximated by a first order Pade approximation.

\subsubsection{Vestibulo Ocular Reflexes (VOR)}

As in its natural counterpart, the human oculomotor reflexes [33], the VOR based on a feedforward control (see figure 5) causes the robot to compensate for movements of two different kinds:

- roll: the rVOR uses the estimated roll angle $\hat{\theta}_{r}$ to compensate for rotation of the body.

- lateral translation: the tVOR based on the estimated robot's linear position $\hat{X}$ minimizes the effects of lateral displacements on the retinal error $\varepsilon_{r}$.

To summarize, the reference angle $\theta_{e r}^{\star}$ (see figure 5) results from the three reflexes (rVOR, tVOR and VFR) as follows:

$$
\theta_{e r}^{\star}=\underbrace{\theta_{e r-\theta_{r}}^{\star}}_{r V O R}+\underbrace{\theta_{e r-X}^{\star}}_{t V O R}+\underbrace{\theta_{e r-V F R}^{\star}}_{V F R}
$$

Figure 7 shows the contribution of the three reflexes during a 1-m imposed lateral displacement with respect to the target (a voluntary movement achieved by changing the set point $X^{\star}$ ) when a 20-cm lateral perturbation (an untoward disturbance corresponding to a gust of wind) was applied to the robot at $t=7 \mathrm{~s}$. It is worth noting that during the imposed translation, the rVOR reacted first (green curve), followed by the VFR (black curve) compensating for the roll variation. In the case of a lateral perturbation without any rotational component, it can be seen, as might be expected, that the VFR reacted faster than the rVOR and tVOR reflexes.

\subsection{The rotational speed controller}

This rotational speed controller makes the robot's follow any change in the rotation speed set points yielded by the attitude-position controller. The propellers are assumed to be driven directly via the thrust. This assumption is not too restrictive because the thrust could be easily obtained thanks:

$$
F_{i}=c_{T} \omega_{i}^{2}
$$



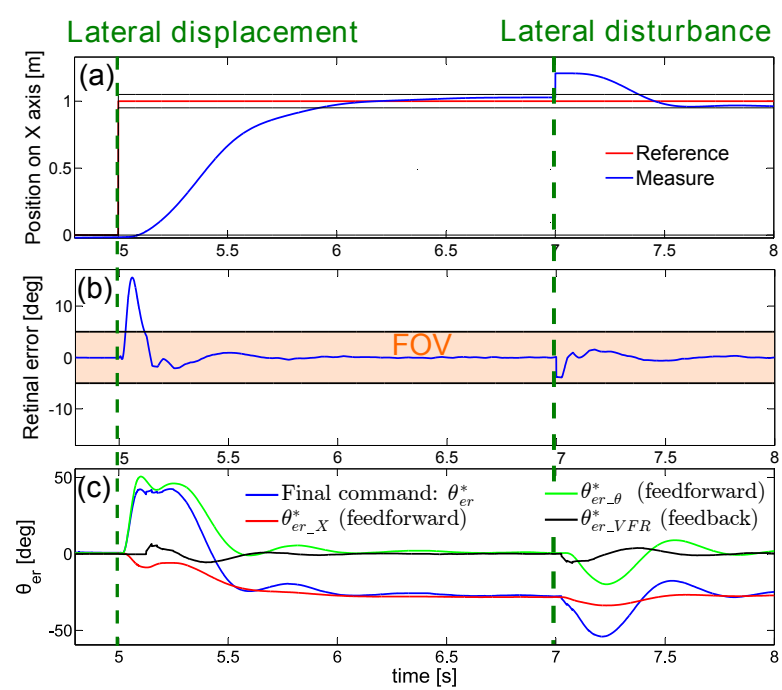
constant $c_{T}$ correspond to the thrust coefficient which could be simply identified using static thrust tests.

This controller consists of a classical controller-observer obtained thanks to the LQ methodology, instead of a PID controller, in order to include the rate gyro's filter dynamics and the motor dynamics. This dynamics are considered to be two first order:

$$
\left(\begin{array}{c}
\dot{\Omega}_{r} \\
\overline{\bar{\Omega}}_{r}
\end{array}\right)=\left(\begin{array}{cc}
\frac{-2 L}{I_{z} \tau_{m o t}} & 0 \\
\frac{1}{\tau_{g y r}} & \frac{-1}{\tau_{g y r}}
\end{array}\right)\left(\begin{array}{c}
\Omega_{r} \\
\bar{\Omega}_{r}
\end{array}\right)+\left(\begin{array}{c}
\frac{2 L}{I_{z} \tau_{m o t}} \\
0
\end{array}\right) \delta^{\star}
$$

where $\Omega_{r}$ is the actual rotational roll speed and $\bar{\Omega}_{r}$ is the rotational speed measured by the rate gyro.

Any static errors occurring in the $\Omega_{r}$ tracking is rejected by an integral effect. Thanks to this controller, the closed loop response time in is less than $20 \mathrm{~ms}$, and the noise in the differential thrust control is less than $2 \%$.

\subsection{Position and attitude controller}

The position and attitude controller were implemented by means of another LQR controller using the states estimated by the nonlinear observer $\left(V_{x}, X\right.$ and $\left.\theta_{r}\right)$. Since 
the dynamics of the rotational speed feedback loop, which have been previously described (see 3.3), were assumed to be much faster than the attitude-position controller, they were neglected.

To design the LQR state feedback, the robot's model (1) was linearised around the origin with the equilibrium control input $\Omega_{r e q}=0$. To cancel any steady state errors, an integral effect was added to the position $X$.

Figure 7 shows a 1-meter displacement along the $X$ axis.

\section{Advantages of the decoupled eye}

The aim of this project is to demonstrate that a decoupled "eye" system presents many benefits contrary to a classical fixed "eye" even if the field of view is restricted to few degrees.

\subsection{Advantage of the VORs}

Figure 8 shows the response of the robot's position to a 1-m reference step input imposed by the setpoint $X^{\star}$ (see figure 5). When the ORs were turned off, only the VFR remained active (the VORs were off). It can be clearly seen from the strong oscillatory response of the robot with no VORs shown in figure 8a and the retinal error shown in figure 8b that the robot with VORs never lost the target (except the initial peak due to the time response of the eye) and showed much smoother and stiffer dynamics than the robot with no VORs. It is worth noting that the robot with no VORs finally succeeded in stabilizing, thanks to the ZSL (see figure 6), which prevented the robot's attitude from becoming unstable when the target was lost.

\subsection{Better disturbances rejection}

In this part we present a comparison of the comportment of two identical robots, one with a fixed eye (denoted F-EYE robot) and the other with the decoupled eye (denoted D-EYE robot). In a first time we will compare two robots with the same FOV, and in a second time, we will assume that the F-EYE robot is equipped with a large FOV $\left( \pm 50^{\circ}\right)$.

\subsubsection{Comparison with a fixed eye robot featuring the same FOV}

The two robots have exactly the same FOV and as a consequence of the limited FOV, the F-EYE robot can not generate aggressive maneuvers. So, this implies that 

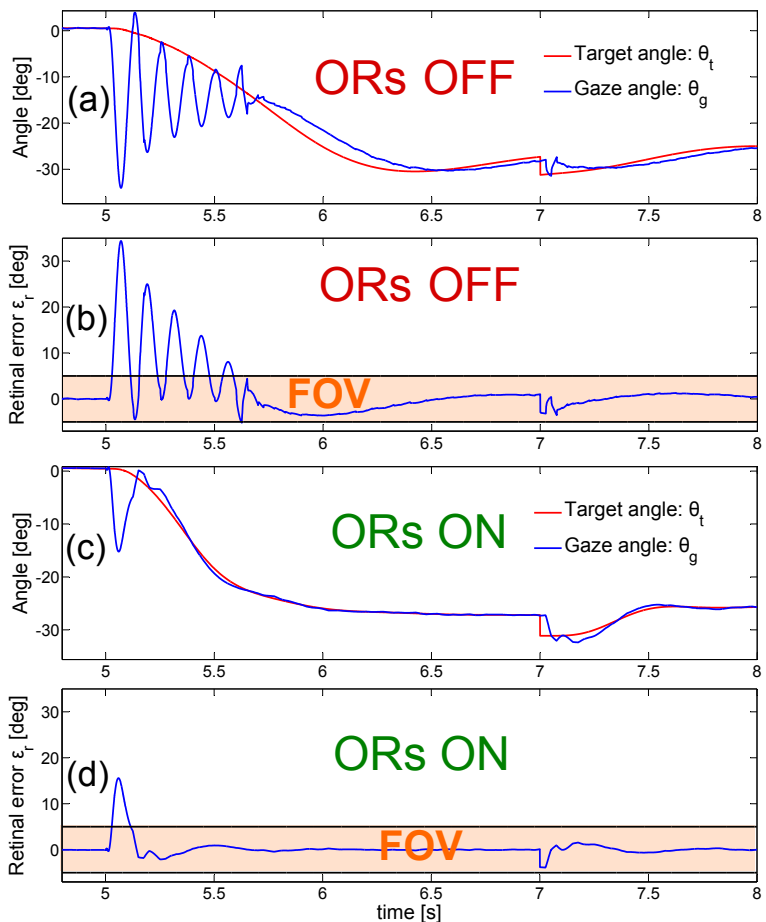

Fig. 8 A 1-m step change in the setpoint $X^{\star}$ led the robot with no ORs (ORs off) to produce a strong oscillatory response in its gaze orientation (a) and thus in the retinal error (b). At $t=7 \mathrm{~s}$ a $20-\mathrm{cm}$ lateral disturbance was applied. Unlike the robot with the ORs switched off, the robot with the ORs switched on can be seen to have rotated its gaze smoothly in order to shift its linear position by $1 \mathrm{~m}$ with respect to the target $(\mathrm{c}$ and d).

the position-controller of the F-EYE robot is less aggressive. Indeed, as it is shown in figure 10a and 10b, when a lateral disturbance appears, it generates a step in the retinal error, allowing to the robot to up to date its position and correct it. But, as the robot is under-actuated, to adjust its position, it is necessary to increase its roll angle which increases again the retinal error. As a consequence, the limit of the FOV is reached for the F-EYE robot, which implies a limitation in roll angle. This limitation implies inevitably a slower rejection of disturbance.

Remark 1. The controller of the D-EYE robot and the F-EYE robot are the same except that the eye controller was removed for the F-EYE robot (as the eye can not rotate). Similarly, the LQ state feedback of the attitude and position controller is quite different. The weighting coefficient of the control input for the F-EYE is 10 times bigger than the one for the D-EYE robot, to avoid going out of FOV. Indeed, to correct its position the robot has to generate roll angle, which implies to increase the retinal error for the F-EYE robot. So, to avoid the target to go out of the FEYE robot's FOV, the position controller is chosen a little less aggressive. This less aggressive controller is achieved by increasing the weighting coefficient of the control input. So we have chosen the lower cost (that is mean the most aggressive comportment), allowing to perform a 1-m displacement without to lose the target for the F-EYE robot.

For the F-EYE and the D-EYE robots, the LQ cost function for the state feedback 


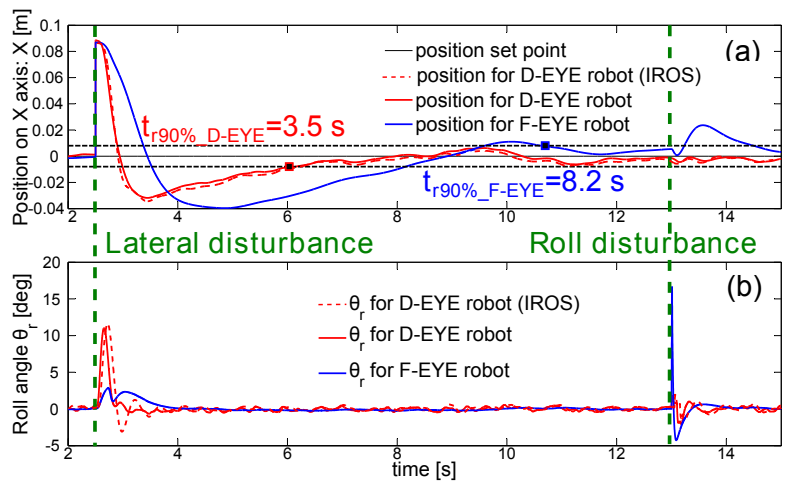

Fig. 9 Responses of the DEYE and F-EYE hovering robots to a 8 -cm lateral disturbance applied at $t=2.5 \mathrm{~s}$ and a strong $20^{\circ}$ rotational disturbance applied at $t=$ $13 \mathrm{~s}$. The robot with a decoupled eye can be seen to have rejected $90 \%$ of the lateral disturbance within $3.5 \mathrm{~s}$, whereas the robot with a fixed eye took $8.2 \mathrm{~s}$ to reject the same disturbance. In b), one can see the improvement with respect to the roll angle provided by adding the retinal error at the observer's input, in comparison to our previous work (IROS).

Fig. 10 Retinal error with the two disturbances described in figure 7 . The retinal error occurring in the case of the robot with a decoupled eye (a) can be seen to have stayed within the limits of the FOV, but (b) reached the limit of the FOV after the lateral perturbation and left the FOV completely for $30 \mathrm{~ms}$ in response to the rotational disturbance of $20^{\circ}$.
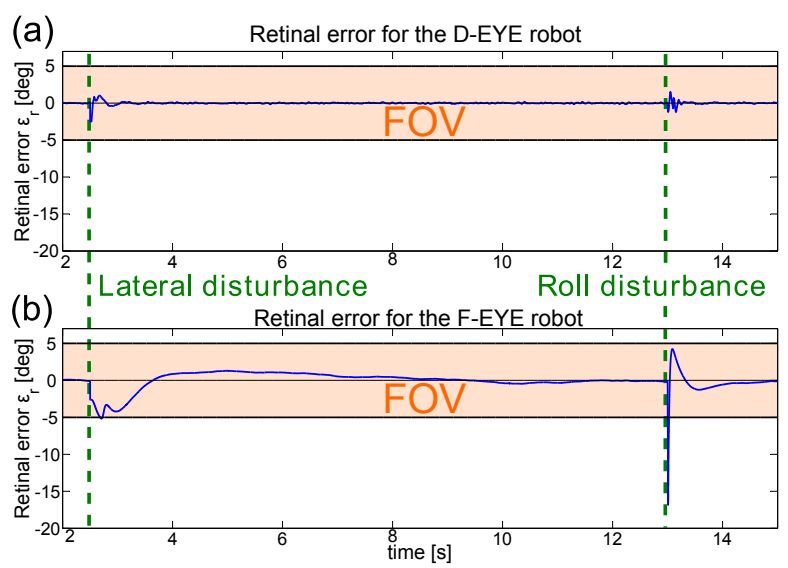

is respectively:

$$
\left\{\begin{array}{l}
J_{F}=\int\left(S^{T} Q_{F} S+\Omega_{r}^{T} R_{F} \Omega_{r}\right) d t \\
J_{D}=\int\left(S^{T} Q_{D} S+\Omega_{r}^{T} R_{D} \Omega_{r}\right) d t
\end{array}\right.
$$

Where $Q_{F}=Q_{D}, R_{F}=10 R_{D}$ and $\mathrm{S}$ the state vector $S=\left(V_{x},\left(X-X^{\star}\right), \theta_{r}, \Delta_{g}\right)^{T}$.

Figure 9 shows the position adopted by the robot and its roll angle in response to a strong lateral $8-\mathrm{cm}$ perturbation (an impulsional perturbation similar to a gust of wind) applied at time $t=2.5 \mathrm{~s}$ and to a $20^{\circ}$ rotational step disturbance around the roll axis applied at $t=13 \mathrm{~s}$. Despite the large lateral disturbance, it can be seen from figure $9 \mathrm{a}$ that the D-EYE robot rejects $90 \%$ of the perturbation within $3.5 \mathrm{~s}$, whereas the F-EYE robot rejects the same perturbation within a much longer period 
of 8.2s. The faster dynamics of the D-EYE robot was obtained at the expense of a much more aggressive control of the roll dynamics (see red curve in figure 9b), while keeping the retinal error within the $\pm 5^{\circ}$ (limits of the FOV visible on figure 10a). This fast rejection is not achievable with the F-EYE robot due to the limit of FOV which are reached (figure 10b). Similarly, the roll disturbance was completely rejected by the D-EYE robot thanks to the ORs, whereas the retinal error left the F-EYE robot's FOV for $30 \mathrm{~ms}$ and it took $1 \mathrm{~s}$ for the error to be completely canceled out.

\subsubsection{Comparison with a fixed eye robot featuring a large FOV}

Visual computational resources for the F-EYE robot

The F-EYE robot is supposed to be equipped with a classical monocular camera with a large FOV of $\pm 50^{\circ}$. Such of camera requires at least 1000 pixels to provide the same resolution as the F-EYE visual sensor $\left(0.1^{\circ}\right)$.

Visual computational resources for the D-EYE robot

The D-EYE robot will be equipped with a custom-made visual sensor endowed with hyperacuity (i.e., the ability to locate a target with a resolution greater than the one composed by the pixel pitch [20]). So, the visual sensor will be composed of few pixels ( 2 pixels), submitted to an active mechanical vibration in order to obtain a resolution as small as $0.1^{\circ}$ for a FOV of only $\pm 5^{\circ}([18]$, [25] and [19]). The visual signal processing is described in [18] and will run on a tiny embedded target (dsPIC, see figure 2).

Simulation results

In the previous part, the controller of the F-EYE robot was less aggressive because of the small FOV and the risk of losing the target for too much roll angle. In this part, we provide to the F-EYE robot a larger FOV of $\pm 50^{\circ}$ than the D-EYE robot. And thanks to this larger FOV, it is possible to have exactly the same controller for the two robots, that is mean that in (6) $Q_{F}=Q_{D}$ and $R_{F}=R_{D}$.

Figures 11 and 12 show the response of the two robots to a strong $15-\mathrm{cm}$ lateral perturbation applied at time $t=3 \mathrm{~s}$ and to a $35^{\circ}$ rotational step disturbance around the roll axis applied at $t=13 \mathrm{~s}$. These two perturbations are bigger than the perturbations applied previously in 4.2.1). It is worth noting that even if the controllers are exactly the same, the D-EYE robot rejects faster the lateral and the roll disturbances than the F-EYE robot. This phenomenon could be explained by the fact that, unlike the F-EYE robot, the D-EYE robot is able to cancel all rotational movements applied on its eye. As a consequence, the D-EYE robot can easily disambiguate a 


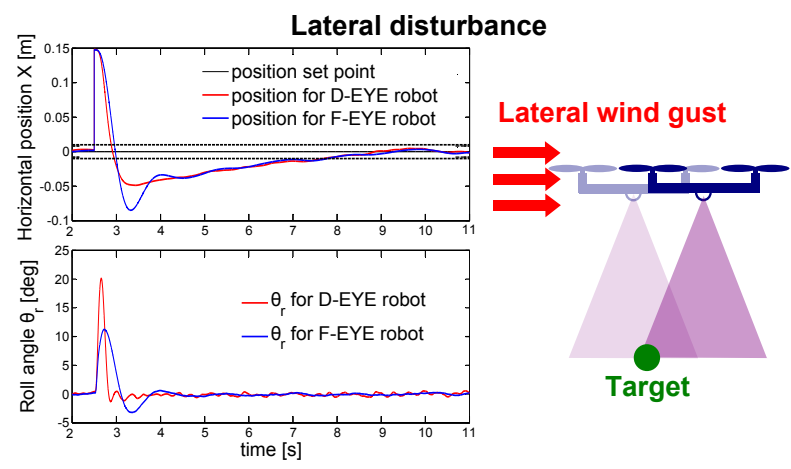

Fig. 11 Response of the DEYE and F-EYE hovering robots to a $15-\mathrm{cm}$ lateral disturbance applied at $t=$ $2.5 s$. The D-EYE robot rejects quiet better the disturbances with a smaller overshoot even if its FOV is only $\pm 5^{\circ}$, while the FOV of the FEYE robot is $50^{\circ}$. This faster rejection is due to the faster convergence of the nonlinear observer.

Fig. 12 Response of the DEYE and F-EYE hovering robots to a strong $35^{\circ}$ roll disturbance applied at $t=$ $13 s$. The D-EYE robot rejects faster the lateral displacement resulting from the roll disturbances with a smaller overshoot.

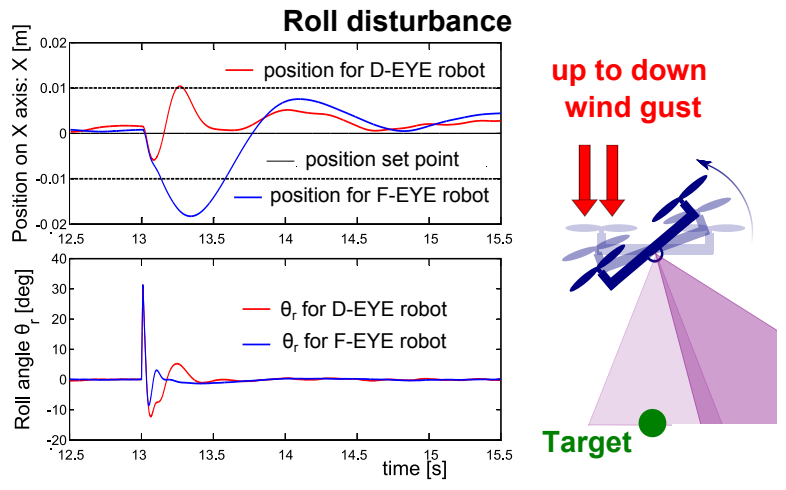

lateral from a rotational disturbance. As a consequence the D-EYE robot provides therefore richer information for the nonlinear observer, which is then able to correct the estimated lateral position with a faster dynamics than the observer of the F-EYE robot. In the figure 11, it can be seen that the D-EYE robot presents a 32-\% overshoot whereas the F-EYE robot response has a 57-\% overshoot. The same phenomenon appears in figure 12 for the roll disturbance where the D-EYE robot has a much more aggressive response in roll and stays close to zero centimeter at $\pm 1.2 \mathrm{~cm}$ whereas the F-EYE robot is much less accurate with a shift in its lateral position of more than $1.9 \mathrm{~cm}$.

\section{Conclusion}

In this paper, a new bio-inspired method of stabilizing an under-actuated hovering aerial robot equipped with a decoupled eye is presented. The results of the simulations performed show that the oculomotor reflexes responsible for stabilizing the gaze and controlling the eye's orientation greatly improve the robot's ability to compensate for strong lateral or rotational (up to $20^{\circ}$ ) disturbances. 
Despite the small field of view of the robot's eye, the new degree of freedom introduced by the decoupled eye easily compensates for this handicap. The fast dynamics of the ORs allowed the robot keep the target within its FOV and served to determine the eye-in-robot's angular position, which unbiased the rate gyro. For this purpose, a new approach to "unbias" the rate gyro was developed, in which the visual loop was used to assess the drift.

In our approach, the accelerometers are replaced by the proprioceptive measurement of the gaze orientation (the eye-in-robot) which plays the same role. In this case, the eye behaves as an inclinometer relative to the target direction. We have shown that the eye is an efficient means to estimate the complete attitude without need of accelerometer. The eye can provide also an accurate and unbiased position estimation of the robot's position, assuming the altitude is known.

The method presented here could be extended in future studies to the stabilization of a robot around the pitch axis. Further research will focus on designing a complete oculomotor system with 3 degrees of freedom and means of stabilizing a hovering autonomous quadrotor with 6 degrees of freedom. However, the use of a decoupled eye goes far beyond the stabilization of a hovering robot, and this development opens promising lines of approach for designing new methods of controlling the 3-D position of a robot by anchoring its gaze on specific objects of interest.

Acknowledgements The authors would like to thank Robert Mahony for fruitful theoretical discussions on the decoupled eye. This work was supported by the CNRS, the University of the Mediterranean and the French National Research Agency (ANR) with the EVA project.

\section{Appendix}

\begin{tabular}{|cccc|}
\hline Parameter & Description & Value & Unit \\
\hline$H$ & Altitude of the hovering robot & 2.0 & $\mathrm{~m}$ \\
$L$ & Half span of the robot & 0.15 & $\mathrm{~m}$ \\
$m$ & Mass of the robot & 0.1 & $\mathrm{~kg}$ \\
$I_{z}$ & Inertia momentum & $2.0 \times 10^{-5}$ & $\mathrm{kg.m}$ \\
$K_{v x}$ & Flapping coefficient & 0.5 & N.s.m \\
$F O V$ & Field of View & \pm 5 & ${ }^{\circ}(\mathrm{deg})$ \\
$d$ & Visual sensor's latency & 10 & $\mathrm{~ms}$ \\
$G_{\text {opt }}$ & Optic sensor's gain & 1 & - \\
$\tau_{\text {hall }}$ & Angle sensor's time constant & 1 & $\mathrm{~ms}$ \\
$\tau_{g y r}$ & Rate gyro's time constant & 4.3 & $\mathrm{~ms}$ \\
$\tau_{\text {mot }}$ & Propeller's time constant & 20 & $\mathrm{~ms}$ \\
$\tau_{\text {eye }}$ & Eye's time constant & 10 & $\mathrm{~ms}$ \\
\hline
\end{tabular}

Table 1 Simulation parameters. 


\begin{tabular}{|c|c|c|c|}
\hline \multicolumn{4}{|c|}{ Sensors } \\
\hline & \begin{tabular}{|l|} 
Visual sensor \\
\end{tabular} & Angle sensor & Rate gyro \\
\hline Transfer function & $G_{\text {opt }}(s)=G_{\text {opt }} e^{-d s}$ & $G_{\text {hall }}(s)=\frac{1}{1+\tau_{\text {hall }}}$ & $G_{g y r}(s)=\frac{1}{1+\tau_{g y r} s}$ \\
\hline Resolution & $0.1\left[^{\circ}\right]$ & $0.1\left[^{\circ}\right]$ & $1\left[{ }^{\circ} \cdot s^{-1}\right]$ \\
\hline Sample frequency & $40[\mathrm{~Hz}]$ & $1[\mathrm{kHz}]$ & $1[\mathrm{kHz}]$ \\
\hline Noise amplitude & $\pm 0.1\left[^{\circ}\right]$ & $\pm 1\left[^{\circ}\right]$ & $\pm 5\left[{ }^{\circ} \cdot s^{-1}\right]$ \\
\hline \multicolumn{4}{|c|}{ Actuators } \\
\hline & Propeller moto & \multicolumn{2}{|c|}{ Eye motor } \\
\hline Transfert function & $G_{m o t}(s)=\frac{1}{1+\tau_{m o t} s}$ & \multicolumn{2}{|c|}{$G_{\text {eye }}(s)=\frac{1}{1+\tau_{\text {eye }} s}$} \\
\hline Rate limiter & - & \multicolumn{2}{|c|}{$1000\left[{ }^{\circ} . s^{-} 1\right]$} \\
\hline
\end{tabular}

Table 2 Sensors and actuators characteristics.

\section{References}

1. A. Manecy, S. Viollet, and N. Marchand, "Bio-Inspired Hovering Control for an Aerial Robot Equipped with a Decoupled Eye and a Rate Gyro," in IEEE/RSJ International Conference on Intelligent Robots and Systems, Vilamoura, Algarve, Portugal, in press, Oct. 2012.

2. J. Wendel, O. Meister, C. Schlaile, and G. F. Trommer, "An integrated gps/mems-imu navigation system for an autonomous helicopter," Aerospace Science and Technology, vol. 10, no. 6, pp. 527 - 533, 2006. [Online]. Available: http://www.sciencedirect.com/science/article/pii/S1270963806000484

3. N. Abdelkrim, N. Aouf, A. Tsourdos, and B. White, "Robust nonlinear filtering for ins/gps uav localization," in Control and Automation, 2008 16th Mediterranean Conference on, june 2008 , pp. $695-702$.

4. A. Nemra and N. Aouf, "Robust ins/gps sensor fusion for uav localization using sdre nonlinear filtering," Sensors Journal, IEEE, vol. 10, no. 4, pp. 789 -798, april 2010.

5. D. Gurdan, J. Stumpf, M. Achtelik, K.-M. Doth, G. Hirzinger, and D. Rus, "Energy-efficient autonomous four-rotor flying robot controlled at $1 \mathrm{khz}$," in Robotics and Automation, 2007 IEEE International Conference on, april 2007, pp. $361-366$.

6. J. Kim, M.-S. Kang, and S. Park, "Accurate modeling and robust hovering control for a quadrotor vtol aircraft," Journal of Intelligent \& Robotic Systems, vol. 57, pp. 9-26, 2010, 10.1007/s10846-009-9369-z.

7. R. Mori, K. Hirata, and T. Kinoshita, "Vision-based guidance control of a small-scale unmanned helicopter," in Intelligent Robots and Systems, 2007. IROS 2007. IEEE/RSJ International Conference on, 29 2007-nov. 2 2007, pp. 2648 -2653.

8. T. Zhang, Y. Kang, M. Achtelik, K. Kuhnlenz, and M. Buss, "Autonomous hovering of a vision/imu guided quadrotor," in International Conference on Mechatronics and Automation, 2009.

9. K. E. Wenzel, P. Rosset, and A. Zell, "Low-cost visual tracking of a landing place and hovering flight control with a microcontroller," in Selected papers from the 2nd international Symposium on UAV, 2009, pp. 297-311.

10. E. Rondon, L.-R. Garcia-Carrillo, and I. Fantoni, "Vision-based altitude, position and speed regulation of a quadrotor rotorcraft," in Conference on Intelligent Robots and Systems, Oct $18-222010$.

11. J. Castellanos, S. Lesecq, N. Marchand, and J. Delamare, "A low-cost air data attitude heading reference system for the tourism airplane applications," in Sensors, 2005 IEEE. IEEE, 2005, pp. 4-pp.

12. S. Weiss, D. Scaramuzza, and R. Siegwart, "Monocular-slam-based navigation for autonomous micro helicopters in gps-denied environments," Journal of Field Robotics, vol. 28, no. 6, pp. 854-874, 2011. [Online]. Available: http://dx.doi.org/10.1002/rob.20412 
13. A. Bachrach, R. He, and N. Roy, "Autonomous flight in unknown indoor environments," International Journal of Micro Air Vehicles, vol. 1756-8293, pp. 217-228, January 2010.

14. S. Grzonka, G. Grisetti, and W. Burgard, "Towards a navigation system for autonomous indoor flying," in Robotics and Automation, 2009. ICRA '09. IEEE International Conference on, may 2009, pp. $2878-2883$.

15. S. Shen, N. Michael, and V. Kumar, "Autonomous multi-floor indoor navigation with a computationally constrained mav," in Robotics and Automation (ICRA), 2011 IEEE International Conference on, may 2011, pp. $20-25$.

16. I. Mondragn, M. Olivares-Mndez, P. Campoy, C. Martnez, and L. Mejias, "Unmanned aerial vehicles uavs attitude, height, motion estimation and control using visual systems," Autonomous Robots, vol. 29, pp. 17-34, 2010. [Online]. Available: http://dx.doi.org/10.1007/s10514-010-9183-2

17. D. H. Ballard, "Animate vision," Artificial Intelligence, vol. 48, no. 1, pp. 57 - 86, 1991.

18. R. Juston and S. Viollet, "A miniature bio-inspired position sensing device for the control of micro-aerial robots," in IEEE/RSJ International Conference on Intelligent Robots and Systems, Vilamoura, Algarve, Portugal, in press, Oct. 2012.

19. S. Viollet and N. Franceschini, "Super-accurate visual control of an aerial minirobot," in Autonomous minirobots for research and edutainment, AMIRE., 2001.

20. G. Westheimer, Visual hyperacuity. Berlin: Ottoson, Sensory Physiology 1, Springer, 1981.

21. S. Viollet and N. Franceschini, "A high speed gaze control system based on the vestibuloocular reflex," Robotics and Autonomous systems, vol. 50, pp. 147-161, 2005.

22. R. Hengstenberg, "Mechanosensorey control of compensatory head roll during flight in the blowfly calliphora erythrocephala meig," Journal of comparative Physiology A, vol. 163, pp. 151-165, 1988.

23. T. S. Collett and M. F. Land, "Visual control of flight behaviour in the hoverflysyritta pipiens 1." Journal of Comparative Physiology A: Neuroethology, Sensory, Neural, and Behavioral Physiology, vol. 99, no. 1, pp. 1-66, Mar. 1975.

24. N. Boeddeker, R. Kern, and M. Egelhaaf, "Chasing a dummy target: smooth pursuit and velocity control in male blowflies," in Proc. R. Soc. Lond.B 270, 2003, pp. 393-399.

25. L. Kerhuel, S. Viollet, and N. Franceschini, "The vodka sensor: A bio-inspired hyperacute optical position sensing device," vol. 12, no. 2, pp. 315-324, 2012.

26. N. Strausfeld, H. Seyan, and J. Milde, "The neck motor system of the fly calliphora erythrocephala. 1. muscles and motor neurons," J. Comp. Physiol, vol. A 160, pp. 205-224, 1987.

27. T. Preuss and R. Hengstenberg, "Structure and kinematics of the prosternal organs and their influence on head position in the blowfly calliphora erythrocephala meig." Journal of Comparative Physiology A: Neuroethology, Sensory, Neural, and Behavioral Physiology, vol. 171, no. 4, pp. 483-493, 1992.

28. J. Zeil, N. Boeddeker, and J. Hemmi, "Vision and the organization of behaviour." Curr Biol, vol. 18, no. 8, pp. R320-R323, Apr 2008.

29. R. Mahony, T. Hamel, and J.-M. Pflimlin, "Nonlinear complementary filters on the special orthogonal group," Automatic Control, IEEE Transactions on, vol. 53, no. 5, pp. $1203-1218$, june 2008 .

30. L. Kerhuel, S. Viollet, and N. Franceschini, "Steering by gazing: An efficient biomimetic control strategy for visually guided micro aerial vehicles," Robotics, IEEE Transactions on, vol. 26 , no. 2, pp. $307-319$, april 2010.

31. D. Robinson, "The mechanics of human smooth pursuit eye movement." The Journal of Physiology, vol. 180, no. 3, pp. 569-591, 1965.

32. L. Stark and L. Young, "Defining biological feedback control systems," Ann N Y Acad Sci, vol. 117, pp. 426-444, Sep 1964.

33. F. Miles, "The neural processing of 3-d visual information: evidence from eye movements," European Journal of Neuroscience, vol. 10, no. 3, pp. 811-822, 1998. 